\title{
Numerical analysis of a thermally tunable spectrally selective absorber enabled by an all-dielectric metamirror
}

\author{
Veysel Erçaglar, ${ }^{1,2, \dagger}$ (1) Volkan Erturk, ${ }^{1,2, *, \dagger}$ Amir Ghobadi, ${ }^{1,2}$ (i) \\ Deniz Umut YILdirim, ${ }^{1,2}$ (1) AND EKmel Ozbay ${ }^{1,2,3,4}$ \\ ${ }^{1}$ NANOTAM-Nanotechnology Research Center, Bilkent University, 06800 Ankara, Turkey \\ ${ }^{2}$ Department of Electrical and Electronics Engineering, Bilkent University, 06800 Ankara, Turkey \\ ${ }^{3}$ Department of Physics, Bilkent University, 06800 Ankara, Turkey \\ ${ }^{4}$ UNAM-Institute of Materials Science and Nanotechnology, Bilkent University, Ankara, Turkey \\ ${ }^{*}$ Corresponding author: volkan.erturk@bilkent.edu.tr
}

Received 7 September 2020; revised 29 September 2020; accepted 16 October 2020; posted 16 October 2020 (Doc. ID 409586); published 9 November 2020

In this Letter, we numerically propose a temperaturetunable, ultra-narrowband one-way perfect near-infrared radiation absorber with high transmission in the longer wavelength neighboring spectral range. We obtained this functionality by using a guided-mode resonance-based grating-waveguide metamirror that is comprised of silicon, a spacer dielectric, an absorbing semiconductor, and germanium. Within the ultra-narrow bandwidth of the guided-mode resonance excited at $1.16 \mu \mathrm{m}$ with a full width at half-maximum of $3.3 \mathrm{~nm}$, we confirmed perfect absorption when light is incident from one of the two opposite directions. Excitation from the opposite direction resulted in perfect reflection. The thickness of the entire structure is limited to about one third the operating wavelength. Furthermore, due to the temperature tunability of silicon and germanium the thermo-optical sensitivity was found to be approximately $0.068 \mathrm{~nm} / \mathrm{K}$. In addition to this spectral tunability, our proposed device supports transparency windows with $80 \%$ transmission in the higher wavelength ranges. Our device is highly promising in the applications of thermo-tunable modulators and obtaining single frequency near-infrared signals from broadband sources. (C) 2020 Optical Society of America

https://doi.org/10.1364/OL.409586

Metamaterials are man-made, i.e., synthetic artificial materials that can achieve optical properties that are not available in nature [1]. In optics, they are used in a wide range of applications such as sensors [2,3], reflectors [4], imaging devices [5], cloaking and invisibility [6], and perfect narrowband [7] or broadband [8] absorbers. Recently, multifunctional metamaterial devices that yield perfect, one-way electromagnetic wave (EMW) absorption in required frequency bands [9] have received much interest in passive terrestrial radiative coolers and single frequency receivers from near-infrared (NIR) sources.
There are several proposed structures that achieve the nearperfect absorption of EM waves such as metal-based plasmonic absorbers and one particular structure that is commonly employed in the literature is the metal-insulator-metal (MIM) cavity architecture [10]. The main disadvantage of this approach is the ohmic losses coming from metal layers that reduce the quality factor of optical cavities and broadens the resonances [11]. In addition to this, using thick metallic reflectors to block the transmission of the radiation in the structure causes the structure to not support transparency and be spectrally selective.

Instead of using optically thick metallic bottom reflectors, by coupling the incident electromagnetic (EM) radiation to the guided modes of the grating structure, perfect reflection is achievable under guided-mode resonance (GMR) conditions [12] for narrow and broadband applications [13]. The thick metallic bottom reflectors of MIM cavities can then be mimicked by these grating structures in a spectrally selective fashion, while simultaneously supporting transparency in the neighboring non-resonant sidebands. Another interesting aspect of grating dielectric grating reflectors is that the spectral position of the excited resonance highly depends on the refractive index of the grating material because it acts as the "core" material of a dielectric slab waveguide $[14,15]$. Therefore, even small changes in the refractive index, which may be induced with temperature, as an example, may cause significant shifts in the position of the excited GMRs [15].

In the present Letter, we make use of the finite-differencetime-domain (FDTD) method to numerically propose an all-dielectric absorber that shows high dynamic tunability by temperature. This functionality is obtained by using Si as a grating buried inside a Si nitride ( $\mathrm{SiN}$ ) layer to build a GMR-based metamirror and adding a germanium (Ge) layer as a lossy material on top. The structure with optimized dimensions behaves as a near-perfect absorber for the NIR radiation, which occurs when the incident radiation is coupled to the guided modes of the grating-waveguide structure. We achieved near-unity absorption at a wavelength of $1160 \mathrm{~nm}$, and the resonance 
is determined to have a full width half-maximum (FWHM) of $3.3 \mathrm{~nm}$. When the radiation is incident from the opposite direction, near-perfect reflection is also verified. Furthermore, thermo-optical sensitivity was found to be $0.068 \mathrm{~nm} / \mathrm{K}$. When NIR radiation is incident from the opposite direction, it behaves as a reflector. In addition to this, deriving this functionality with an all-dielectric structure provides transparency, i.e., high transmission in the lower energy spectral bands neighboring the resonant window. The thickness of the overall structure is also limited to the sub-wavelength range, only $395 \mathrm{~nm}$, as opposed to the operation wavelength of $1160 \mathrm{~nm}$.

Our proposed structure in Figs. 1(a) and 1(b) consists of a $\mathrm{Si}$ frequency-selective reflector, SiN spacer, and Ge absorbing layer. It is designed in such a way to give different responses when excited from the top or bottom. These two types of excitations are defined as follows: when the incoming radiation is incident from the $\mathrm{Ge}(\mathrm{Si})$ side and travels in the $-z(+z)$ direction of Fig. 1(a), it is defined as forward (backward) excitation.

We utilize FDTD simulations to scrutinize the spectral response of our proposed device. In the simulations, we illuminated the unit cell with a broadband plane-wave at normal incidence whose electric field (E-field) is polarized in the $x$ direction $(\phi=0, p$ polarization). In the $x$ and $y$ directions of the simulation region, we employed periodic boundary conditions, while in the $z$ direction perfectly matched layers (PML) are adopted. We use two monitors to find the reflected $(R)$ and transmitted $(T)$ power, normalized to the incident power. The dispersion effect of $\mathrm{Si}, \mathrm{SiN}$, and $\mathrm{Ge}$ are retrieved with ellipsometry measurements at $273 \mathrm{~K}$, and they were observed to be in good agreement with the models based on the Sellmeier equation [16].

We started our analysis of the proposed design by setting the geometrical dimensions outlined in Fig. 1(a) to their optimized values: thickness of Si grating stripes, $h_{\mathrm{Si}}=260 \mathrm{~nm}$; SiN thickness above and below $\mathrm{Si}, h_{\mathrm{SiN}}=50 \mathrm{~nm}$; Ge layer thickness, $h_{\mathrm{Ge}}=30 \mathrm{~nm}$; grating stripe width, $W=550 \mathrm{~nm}$; unit-cell periodicity, $L=975 \mathrm{~nm}$. We then simulated this structure to find $R$ and $T$ and passed it to the absorption $(A)$ by using the formula $A=1-R-T$. The spectral response of this device, under forward and backward illumination cases, is shown in the top and bottom subfigures of Fig. 1(c), respectively.
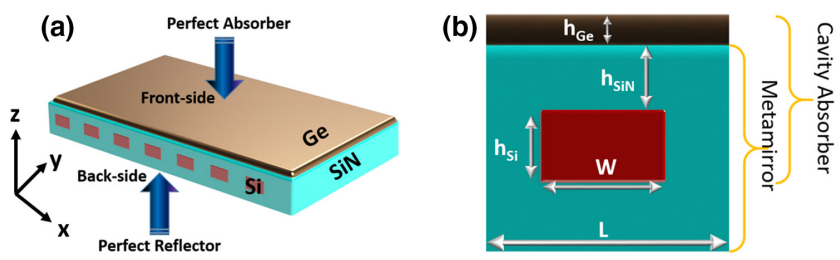

(c)

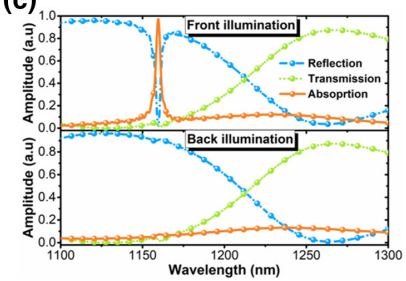

Fig. 1. (a) Three-dimensional (3D) layout of the structure. (b) Twodimensional (2D) front view of the structure, scrutinizing the unit cell. Spectral response of the proposed device under (c) forward and backward illumination cases.
Figure 1(c) shows a sharp GMR that peaks at $1160 \mathrm{~nm}$, with a FWHM of $3.3 \mathrm{~nm}$. This figure also proves the asymmetric reflection/absorption property of our device with transparency in the longer wavelength regimes. This asymmetry is because of the fact that under forward excitation and within the bandwidth of this GMR, the Si grating effectively acts as a mirror, and, with the addition of a SiN spacer and Ge absorbing layers, the structure mimics a Fabry-Perot-type cavity absorber. In the backward illumination case, however, the grating directly reflects the incident radiation before it can reach the Ge layer to be harnessed there.

Excitation of the GMR, which gives our device our asymmetric absorption/reflection functionality, with transparency functionality that stems from the fact that the Si grating, buried inside the SiN layer, also acts a waveguide core, if a diffracted order is phase-matched to a guided mode of the waveguide core. For a one-dimensional (1D) grating, as in this work, this physical phenomenon is governed by Eq. (1) [12]:

$$
k_{x, g}=m K_{x}=m \frac{2 \pi}{\Lambda_{x}}=m \frac{2 \pi}{L}, \beta=\left|k_{x, g} \hat{\boldsymbol{x}}\right|,
$$

where $\beta$ is the propagation wavenumber along the guiding direction, $\Lambda_{x}\left(K_{x}\right)$ is the grating period (reciprocal lattice vector) along the $x$ direction. $m$ is the diffraction order along the $x$ direction, and $k_{x, g}$ is the wavevector component of the guided mode along the $x$ direction. As the guided mode propagates, it also continually leaks energy to the far field because of the periodicity of the waveguide core. The sharp increase in the reflection is because the re-radiated waves destructively interfere with the directly $T$ waves, but constructively with the $\mathrm{R}$ waves.

To further support this result, we use symmetric dielectric waveguide theory, where the core material is the $\mathrm{Si}-\mathrm{SiN}$ hybrid, and the cladding materials are SiN. For practicality in equations, we assume that cladding layers extend well beyond the evanescent decay length of the guided mode. The effective refractive index of the core material is calculated by adopting Rytov's formula in Eq. (2) [17], for the case of the E-field being perpendicular to the grating grooves:

$$
\begin{gathered}
\frac{1}{n_{\text {core }}^{2}}=f \frac{1}{n_{\mathrm{Si}}^{2}}+(1-f) \frac{1}{n_{\mathrm{SiN}}^{2}}, \\
\tan \left(h t_{\mathrm{core}}\right)=\frac{2 \bar{p}}{h\left(1-\frac{\bar{p}^{2}}{h^{2}}\right)},
\end{gathered}
$$

where $f$ is the fill factor for the grating, which is $W / L$ in our case. Then, because the incident magnetic field ( $\mathrm{H}$-field) is polarized transverse to the grating vector, we investigated the TM-mode excitation in a symmetric dielectric waveguide, the transcendental equation for which is given in Eq. (3) [14]. In this equation, $b=k_{0} \sqrt{n_{\text {core }}^{2}-n_{\text {eff }}^{2}}$ is the transverse component of the wavevector in the core, $\bar{p}=n_{\text {core }}^{2} / n_{\text {SiN }}^{2} * k_{0} \sqrt{n_{\text {eff }}^{2}-n_{\text {SiN }}^{2}}$ is the decay constant of the guided mode in the cladding. Then, the right-hand and left-hand side (RHS and LHS, respectively) of Eq. (3) is plotted, along with Eq. (1), with $m=2$ value, and their intersection is observed in Fig. 2. The $\beta$ value coming from the reciprocal lattice vector is slightly off from the intersection of RHS and LHS. This is because $h_{\mathrm{SiN}}$ is picked thin for reasons that will be explained later, so the actual structure differs from 


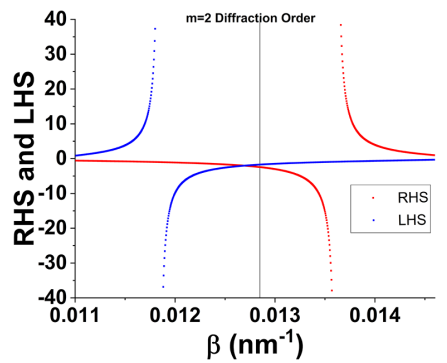

Fig. 2. Quantitaive analysis of the excited GMR.

the infinite cladding model. Therefore, although Eq. (3) gives a firm starting point to investigate mode excitation, it is not totally exact for our case.

Geometrical parameter adjustment also gives our design a wide range of tunability, in terms of the resonant window. By adjusting the geometrical parameters of the Si grating, buried inside the SiN material, we were able to adjust the spectral position of peak reflection, as can be seen in Figs. 3(a) and 3(b), for changing $h_{\mathrm{Si}}$ and $W$. In Fig. 3(a), we sweep $h_{\mathrm{Si}}$, while keeping the other parameters in their aforementioned values. We observed that as $h_{\mathrm{Si}}$ is increased, the existing mode red shifts, which is an expected result from the dielectric waveguide theory because modes are supported above a certain normalized core thickness, $h_{\mathrm{Si}} / \lambda$, so an increase in the former also increases the (a)

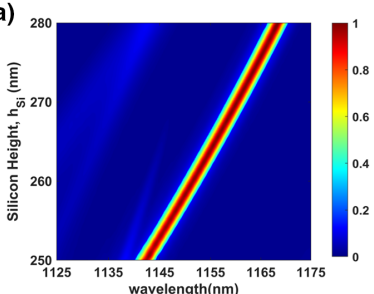

(c)

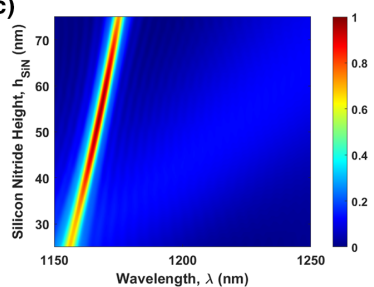

(e)

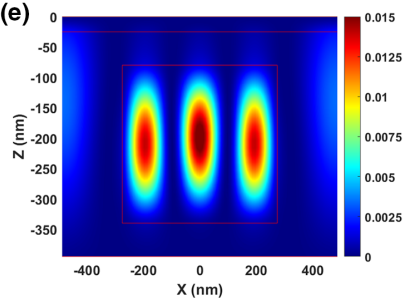

(b)

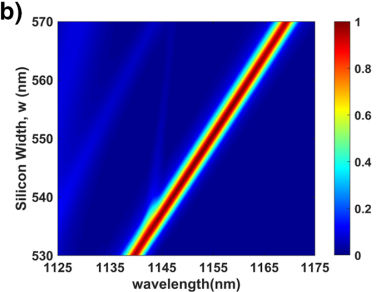

(d)

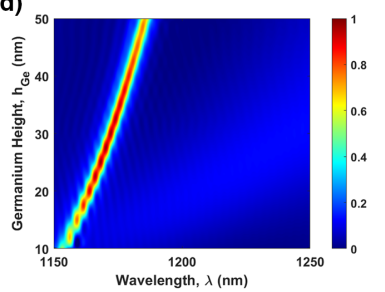

(f)

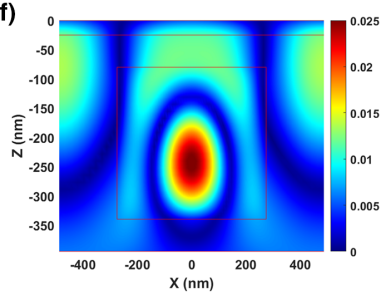

Fig. 3. Absorption spectra for (a) thickness of Si layer versus wavelength when the width of the Si layer is kept constant at $550 \mathrm{~nm}$ and (b) width of Si layer versus wavelength when thickness of the Si layer is kept constant at $260 \mathrm{~nm}$. Absorption spectra for (c) thickness of SiN layer versus wavelength when thickness of the Ge layer is kept constant at $35 \mathrm{~nm}$ and (d) thickness of Ge layer versus wavelength when the thickness of the SiN layer is kept constant at $300 \mathrm{~nm}$. At resonant wavelength, $\mathrm{H}$-field magnitude $\left(|H|^{2}\right)$ profile at the $\mathrm{XZ}$ plane under (e) forward excitation and (f) backward excitation conditions that are being scrutinized. latter [14]. In Fig. 3(b), $W$ is swept while the other parameters are kept constant. It is observed that, with increasing $W$, the waveguide mode again red shifts because the waveguide effective core refractive index increases due to the larger filling factor of $\mathrm{Si}$. It is also critical to mention that GMRs can give both a narrowband and broadband behavior depending on the confinement of the mode to the waveguide core and the spectral overlap between distinct modes [13]. For our aim of realizing a dynamically tunable device, we designed our reflector to operate in one of the narrowband modes because a narrow linewidth increases the modulation depth and, consequently, the figure-of-merit [18] in a modulator, which is another potential application of our proposed device. We also swept $h_{\mathrm{SiN}}$, while keeping the other parameters constant, and the result is outlined in Fig. 3(c). It is seen that $h_{\mathrm{SiN}}$ has an optimum value for perfect absorption because it creates the necessary phase accumulation to redirect the EMWs mostly to the top absorbing layer. Next, $h_{\mathrm{Ge}}$ is swept, the results of which are depicted in Fig. 3(d). It is seen that a thick layer of $\mathrm{Ge}$ cannot give perfect absorption because it decouples the cavity from the incoming radiation, due to not letting the radiation effectively reach the cavity. On the other hand, as $h_{\mathrm{Ge}}$ decreases too much, the radiation that is re-directed to this layer cannot be harnessed and leaks out as radiation. It is noteworthy that GMRs red shift when $h_{\mathrm{Ge}}$ and $h_{\mathrm{SiN}}$ increase. This is because their thickness is picked less than the evanescent decay length of the guided mode in the cladding region, i.e., the $\mathrm{SiN}-\mathrm{Ge}$ combination, so the optical fields in these layers are not small enough to be neglected, and they necessitate new boundary conditions. Therefore, $\beta$ and the GMR peak wavelength are modified compared to an infinite cladding dielectric waveguide [13]. Due to this, the optimization of the structure is performed holistically by having all layers present in the simulation, instead of first optimizing the metamirror and then adding the Ge layer. Thicker layers of $\mathrm{SiN}$ could be picked to make the GMR wavelength insensitive to variations in $h_{\mathrm{Ge}}$ and $h_{\mathrm{SiN}}$, but it would make the device thicker and thereby bulkier. It is also worth mentioning that dielectric spacers other than SiN could also be used, such as $\mathrm{Si}$ dioxide $\left(\mathrm{SiO}_{2}\right)$ and Magnesium Fluoride $\left(\mathrm{MgF}_{2}\right)$. The reason for picking SiN is that GMR linewidth is proportional to the difference between the permittivities of core material and surrounding medium [12], so using SiN results in a smaller linewidth compared to the other two. Compared to other high-refractive index materials like $\mathrm{TiO}_{2}$, as an example, it provides better adhesion to $\mathrm{Si}$, so it makes experimental verification more practicable.

To further discern the resonance behavior, we also plotted the H-field in resonance conditions for forward and backward excitations, the results of which are shown in Figs. 3(e) and $3(\mathrm{f})$, respectively. Comparison between these figures indicate two orders of magnitude enhancement $\mathrm{H}$-field and, more importantly, localization in the Si waveguide core. Importantly, Fig. 3(e) shows that the third-order mode of the waveguide is excited. The reason for this choice of ours is that higher-order modes, compared to the fundamental mode, can give smaller linewidths due to being closer to the mode cutoff [19]. However, these higher-order modes also necessitate a larger core thickness, $h_{\mathrm{Si}}$, to be supported. We, therefore, used the third-order mode as it gives a good trade-off between these two concerns. Finally, the structure has been simulated for various temperatures. Changes in temperature bring changes in the refractive index of materials and their dimensions. However, changes in dimensions are too 
small compared to the overall structure, i.e., on the order of $10^{-4} \mathrm{~nm}$ [20]. Therefore, our focus has been on the changes in the dielectric permittivity of the materials, especially $\mathrm{Si}$ and $\mathrm{Ge}$, to dynamically tune our device. To model the permittivity of the materials as a function of temperature, temperature dependent Sellmeier's coefficients [16] are used to calculate the refractive indices of the materials, as given in Eq. (4):

$$
\begin{aligned}
& n^{2}(\lambda, T)-1=\sum_{i}^{n} \frac{S_{i}(T) \cdot \lambda^{2}}{\lambda^{2}-\lambda_{i}^{2}(T)}, \\
& \quad S_{i}(T)=\sum_{j}^{m} S_{i j}(T) \cdot T^{j}, \lambda_{i}(T)=\sum_{j}^{m} \lambda_{i j}(T) \cdot T^{j} .
\end{aligned}
$$

Although the refractive index change of Si and Ge with temperature may be approximated as a linear increase, the method above gives more accurate refractive index values. The thermo-optic coefficient $(d n / d T)$ of Si and Ge were found to be approximately $1.9 \times 10^{-4} \mathrm{~K}^{-1}$ and $3 \times 10^{-3} \mathrm{~K}^{-1}$, respectively, between $273 \mathrm{~K}$ and $473 \mathrm{~K}$ at operating wavelengths [21]. SiN, however, has very small temperature dependency of the refractive index. As the temperature increases, an absorption peak has shifted to longer wavelengths without a change in the absorption amplitude, which is depicted with visible radiation for demonstration purposes in Fig. 4(a) and numerically in Fig. 4(b). Next, a straight line for the set of resonance peak $\lambda_{\max }$ values with respect to the applied temperature is generated by utilizing the linear polynomial fitting function of MATLAB, and, from the slope of this line, the thermo-optical sensitivity of the structure was found to be $0.068 \mathrm{~nm} / \mathrm{K}$, which is shown in Fig. 4(c). The equation for this line is $\lambda_{\max }=0.068 T+1138.87$. While the thermo-optic response of $\mathrm{Si}$ modifies the core refractive index of the core, the response of Ge modifies that of the cladding, therefore modifying $\beta$, and thereby the peak GMR wavelength. This is another reason as to why we picked $h_{\mathrm{SiN}}$ thin. By this way,

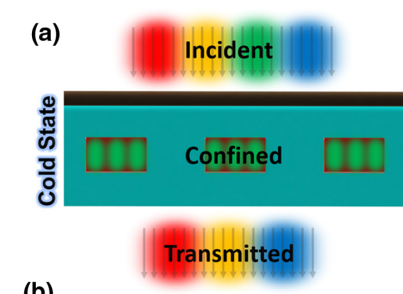

(b)

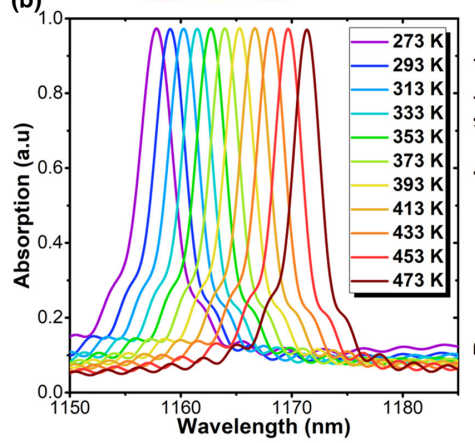

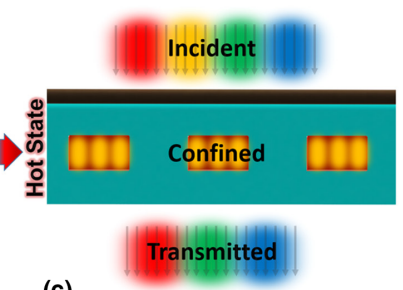

(c)

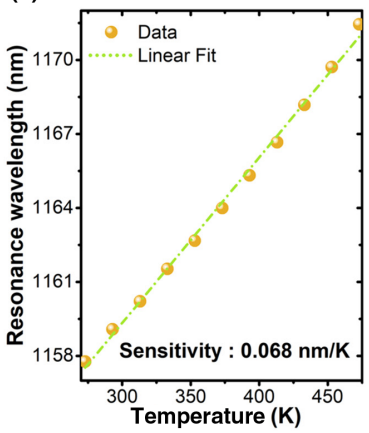

Fig. 4. (a) Illustration of red shift of the absorption peak as the temperature is increased. (b) Absorption spectrum change with temperature. (c) Resonance wavelengths for different applied temperature values and linear fit in order to find the thermo-optical sensitivity of the proposed device.
Ge was able to contribute to the thermo-optical sensitivity by being able to influence $\beta$, because otherwise the guided mode would decay a lot before interacting with the Ge layer, i.e., only SiN would be influencing $\beta$.

Experimental verification of this work can be performed by patterning the Si layer with electron-beam lithography and then deposition of the other dielectric layers. The characterization can be done by using a Fourier transform infrared spectroscopy setup.

In conclusion, in this letter, we proposed a metamaterial structure that achieves thermally tunable narrowband absorption from one side and reflection from the other side, while supporting transparency in the higher-wavelength range. In our final design, the device had perfect absorption with a resonance linewidth of $3.3 \mathrm{~nm}$ and a thermo-optical sensitivity of $0.068 \mathrm{~nm} / \mathrm{K}$.

Funding. Türkiye Bilimsel ve Teknolojik Araştirma Kurumu (113E331, 114E374, 115F560, 116F438, 118E397); Türkiye Bilimler Akademisi.

Disclosures. The authors declare no conflicts of interest.

${ }^{\dagger}$ These authors contributed equally to this Letter.

\section{REFERENCES}

1. N. Engheta and R. W. Ziolkowski, Metamaterials: Physics and Engineering Explorations (Wiley-Interscience, 2006).

2. D. U. Yildirim, A. Ghobadi, M. C. Soydan, M. Gokbayrak, A. Toprak, B. Butun, and E. Ozbay, J. Phys. Chem. C 123, 19125 (2019).

3. M. C. Soydan, A. Ghobadi, D. U. Yildirim, E. Duman, A. Bek, V. B. Erturk, and E. Ozbay, Adv. Opt. Mater. 8, 1901203 (2020).

4. J. Zhang, S. Shi, H. Jiao, X. Ji, Z. Wang, and X. Cheng, Photon. Res. 8, $426(2020)$.

5. S. Ogawa and M. Kimata, Materials 10, 493 (2017).

6. F. Monticone, R. Fleury, and A. Alu, in USNC-URSI Radio Sci. Meet. (Joint with AP-S Symp.) (USNC-URSI, 2013).

7. D. U. Yildirim, A. Ghobadi, and E. Ozbay, Sci. Rep. 8, 1 (2018).

8. D. U. Yildirim, A. Ghobadi, M. C. Soydan, O. Atesal, A. Toprak, M. D. Caliskan, and E. Ozbay, ACS Photon. 6, 1812 (2019).

9. M. Mutlu, S. Cakmakyapan, A. E. Serebryannikov, and E. Ozbay, Phys. Rev. B 87, 205123 (2013).

10. K. Aydin, V. E. Ferry, R. M. Briggs, and H. A. Atwater, Nat. Commun. 2, 517 (2011).

11. Z. Li, S. Butun, and K. Aydin, ACS Photon. 2, 183 (2015).

12. D. Rosenblatt, A. Sharon, and A. A. Friesem, IEEE J. Quantum Electron. 33, 2038 (1997).

13. D. U. Yildirim, A. Ghobadi, M. C. Soydan, A. E. Serebryannikov, and E. Ozbay, Opt. Lett. 45, 2010 (2020).

14. A. Yariv and P. Yeh, Photonics: Optical Electronics in Modern Communications, The Oxford Series in Electrical and Computer Engineering (Oxford University, 2006).

15. M. J. Uddin and R. Magnusson, IEEE Photon. Technol. Lett. 25, 1412 (2013).

16. E. D. Palik, Handbook of Optical Constants of Solids (Academic, 2012).

17. S. M. Rytov, Sov. Phys. JETP 2, 466 (1956).

18. M. Jacques, A. Samani, E. El-Fiky, D. Patel, Z. Xing, and D. V. Plant, Opt. Express 27, 10456 (2019).

19. S. S. Wang and R. Magnusson, Appl. Opt. 32, 2606 (1993).

20. H. Watanabe, N. Yamada, and M. Okaji, Int. J. Thermophys. 25, 221 (2004).

21. B. J. Frey, D. B. Leviton, and T. J. Madison, Proc SPIE 6273, 62732J (2006). 